\title{
Asymptotic behavior of Pascal's triangle modulo a prime
}

\author{
by \\ BRAD WiLson (Brockport, N.Y.)
}

1. Definitions and notation. Let $p$ be a prime and let $F_{p}(n)$ denote the number of entries in the first $n$ rows of Pascal's triangle not divisible by $p$. In 1947 Fine [1] showed

Theorem (Fine, 1947).

$$
\lim _{n \rightarrow \infty} F_{p}(n) /\left(\begin{array}{c}
n+1 \\
2
\end{array}\right)=0 .
$$

Fine made use of the well-known result of Kummer [3]:

THEOREM (Kummer, 1852). The highest power of a prime $p$ that divides $\left(\begin{array}{l}n \\ m\end{array}\right)$ is equal to the number of carries that occur when adding $m$ and $n-m$ in base $p$.

The following is a well-known corollary of Kummer's theorem which we will need.

COROLlary (of Kummer's Theorem). The number of entries in the nth row of Pascal's triangle not divisible by $p$ is

$$
2^{r_{1}} 3^{r_{2}} \ldots p^{r_{p-1}}
$$

where $r_{i}=\left|\left\{j: n_{j}=i\right\}\right|$ for $n=\left(n_{k} n_{k-1} \ldots n_{1} n_{0}\right)_{p}$ is the number of $i$ 's in the base $p$ expansion of $n$.

Let $\theta=\ln 3 / \ln 2$ and

$$
\alpha=\limsup _{n \rightarrow \infty} F_{2}(n) / n^{\theta}, \quad \beta=\liminf _{n \rightarrow \infty} F_{2}(n) / n^{\theta} .
$$

In 1977 Harborth [2] showed

Theorem (Harborth, 1977). $\alpha=1$ and $\beta=.812556 \ldots$

1991 Mathematics Subject Classification: 11B65, 11B50, 05A10.

Key words and phrases: Pascal's triangle. 
The value of $\beta$ was determined to six decimal places and Harborth conjectured that $\beta=\lim _{r \rightarrow \infty} q_{r}$ for $q_{r}=F\left(n_{r}\right) / n_{r}^{\theta}$ where $n_{0}=1, n_{i}=n_{i-1} \pm 1$ with sign chosen to minimize $F\left(n_{i}\right) / n_{i}^{\theta}$.

More generally, let $\theta_{p}=\ln (p(p+1) / 2) / \ln p$ and

$$
\alpha_{p}=\limsup _{n \rightarrow \infty} F_{p}(n) / n^{\theta_{p}}, \quad \beta_{p}=\liminf _{n \rightarrow \infty} F_{p}(n) / n^{\theta_{p}} .
$$

In 1989 Stein [4] proved

Theorem (Stein, 1989). $\alpha_{p}=1$.

In this paper we calculate $\beta_{3}, \beta_{5}, \ldots, \beta_{19}$ to six decimal places, get upper and lower bounds on $\beta_{p}$ which allow us to show $\lim _{p \rightarrow \infty} \beta_{p}=.5$, and expand on Harborth's conjecture.

2. Lemmas on the behavior of $F_{p}(n)$. We first note some elementary facts about $F_{p}(n)$.

Lemma 1. For $1 \leq a \leq p-1$ and $0 \leq b \leq p^{r}$,

$$
F_{p}\left(a p^{r}+b\right)=\frac{a(a+1)}{2} F_{p}\left(p^{r}\right)+(a+1) F_{p}(b) .
$$

Proof. This is a corollary of [4], Lemma 3.

Lemma 1 allows us to prove that $F_{p}(n)=n^{\theta_{p}}$ for infinitely many wellchosen $n$ :

Lemma 2. $F_{p}\left(p^{r}\right)=(p(p+1) / 2)^{r}=\left(p^{r}\right)^{\theta_{p}}$ for all $r \in \mathbb{N}$.

Proof. This was shown in [1].

Lemma 3. For $p$ a prime and $n, k \in \mathbb{N}$,

$$
F_{p}\left(p^{k} n\right)=\left(\frac{p(p+1)}{2}\right)^{k} F_{p}(n),
$$

and

$$
\frac{F_{p}\left(p^{k} n\right)}{\left(p^{k} n\right)^{\theta_{p}}}=\frac{F_{p}(n)}{n^{\theta_{p}}}
$$

Proof. This is a corollary of [4], Lemma 3 .

LEMMA 4. For $n=a_{k} p^{k}+a_{k-1} p^{k-1}+\ldots+a_{1} p+a_{0}, a_{k} \neq 0,0 \leq a_{i}<p$ we have

$$
\begin{aligned}
F_{p}\left(p^{k}+n\right) & \\
= & \begin{array}{ll}
\frac{2+a_{k}}{2}\left(\frac{p(p+1)}{2}\right)^{k}+\frac{2+a_{k}}{1+a_{k}} F_{p}(n) & \text { if } a_{k} \neq p-1, \\
\left(\frac{p(p+1)}{2}\right)^{k+1}-(p-1)\left(\frac{p(p+1)}{2}\right)^{k}+\frac{2}{p} F_{p}(n) & \text { if } a_{k}=p-1 .
\end{array}
\end{aligned}
$$


Proof. If $a_{k}<p-1$ then $1+a_{k}<p$ so

$$
F_{p}\left(p^{k}+n\right)=F_{p}\left(\left(1+a_{k}\right) p^{k}+a_{k-1} p^{k-1}+\ldots+a_{1} p+a_{0}\right) .
$$

By Lemma 1 this becomes

$$
\begin{aligned}
& \frac{\left(2+a_{k}\right)\left(1+a_{k}\right)}{2} F_{p}\left(p^{k}\right)+\left(2+a_{k}\right) F_{p}\left(a_{k-1} p^{k-1}+\ldots+a_{1} p+a_{0}\right) \\
= & \frac{2+a_{k}}{2} F_{p}\left(p^{k}\right)+\frac{a_{k}\left(2+a_{k}\right)}{2} F_{p}\left(p^{k}\right) \\
& +\left(2+a_{k}\right) F_{p}\left(a_{k-1} p^{k-1}+\ldots+a_{1} p+a_{0}\right) \\
= & \frac{2+a_{k}}{2} F_{p}\left(p^{k}\right) \\
& +\frac{2+a_{k}}{1+a_{k}}\left(\frac{a_{k}\left(1+a_{k}\right)}{2} F_{p}\left(p^{k}\right)+\left(1+a_{k}\right) F_{p}\left(a_{k-1} p^{k-1}+\ldots+a_{1} p+a_{0}\right)\right) .
\end{aligned}
$$

Using Lemmas 1 and 2 this is equal to

$$
\begin{array}{r}
\frac{2+a_{k}}{2}\left(\frac{p(p+1)}{2}\right)^{k}+\frac{2+a_{k}}{1+a_{k}} \\
F_{p}\left(a_{k} p^{k}+a_{k-1} p^{k-1}+\ldots+a_{1} p+a_{0}\right) \\
=\frac{2+a_{k}}{2}\left(\frac{p(p+1)}{2}\right)^{k}+\frac{2+a_{k}}{1+a_{k}} F_{p}(n) .
\end{array}
$$

If $a_{k}=p-1$ then $1+a_{k}=p$ so

$$
F_{p}\left(p^{k}+n\right)=F_{p}\left(p^{k+1}+a_{k-1} p^{k-1}+\ldots+a_{1} p+a_{0}\right),
$$

which by Lemma 1 is

$$
F_{p}\left(p^{k+1}\right)+2 F_{p}\left(a_{k-1} p^{k-1}+\ldots+a_{1} p+a_{0}\right) .
$$

With some algebraic manipulation we get

$$
\begin{aligned}
& F_{p}\left(p^{k+1}\right)-(p-1) F_{p}\left(p^{k}\right)+(p-1) F_{p}\left(p^{k}\right)+\frac{2}{p} \cdot p F_{p}\left(a_{k-1} p^{k-1}+\ldots+a_{1} p+a_{0}\right) \\
& =F_{p}\left(p^{k+1}\right)-(p-1) F_{p}\left(p^{k}\right) \\
& \quad+\frac{2}{p}\left(\frac{p(p-1)}{2} F_{p}\left(p^{k}\right)+p F_{p}\left(a_{k-1} p^{k-1}+\ldots+a_{1} p+a_{0}\right)\right) \\
& =F_{p}\left(p^{k+1}\right)-(p-1) F_{p}\left(p^{k}\right)+\frac{2}{p} F_{p}\left((p-1) p^{k}+a_{k-1} p^{k-1}+\ldots+a_{1} p+a_{0}\right) \\
& =\left(\frac{p(p+1)}{2}\right)^{k+1}-(p-1)\left(\frac{p(p+1)}{2}\right)^{k}+\frac{2}{p} F_{p}(n) .
\end{aligned}
$$

The next lemma says that if we have a lower bound on $F_{p}(n) / n^{\theta_{p}}$ for $n$ with lead coefficient $a_{k}$ then we get a lower bound on the quotient where the lead coefficient of $n$ is incremented by one. The new bound depends only 
on the value of the lead coefficient and not on $k$ (i.e. the power of $p$ it is a coefficient of).

Lemma 5. For $1 \leq a_{k}<p-1$ fixed, if there is a constant $\gamma_{p}\left(a_{k}\right)$ so that

$$
\frac{F_{p}(n)}{n^{\theta_{p}}}>\gamma_{p}\left(a_{k}\right)
$$

for all $n=a_{k} p^{k}+a_{k-1} p^{k-1}+\ldots+a_{1} p+a_{0}$, then

$$
\frac{F_{p}\left(p^{k}+n\right)}{\left(p^{k}+n\right)^{\theta_{p}}}>\frac{2+a_{k}}{2}\left(1+\left(\frac{1+a_{k}}{2 \gamma_{p}\left(a_{k}\right)}\right)^{1 /\left(\theta_{p}-1\right)}\right)^{1-\theta_{p}},
$$

i.e., we may take $\gamma_{p}\left(1+a_{k}\right)$ as the right hand side of the inequality.

Pr o of. By Lemma 4 we have

$$
\frac{F_{p}\left(p^{k}+n\right)}{\left(p^{k}+n\right)^{\theta_{p}}}=\frac{\frac{2+a_{k}}{2}\left(\frac{p(p+1)}{2}\right)^{k}+\frac{2+a_{k}}{1+a_{k}} F_{p}(n)}{\left(p^{k}+n\right)^{\theta_{p}}} .
$$

Using our assumption $F_{p}(n)>n^{\theta_{p}} \gamma_{p}\left(a_{k}\right)$ we get

$$
\frac{F_{p}\left(p^{k}+n\right)}{\left(p^{k}+n\right)^{\theta_{p}}}>\frac{\frac{2+a_{k}}{2}\left(\frac{p(p+1)}{2}\right)^{k}+\frac{2+a_{k}}{1+a_{k}} n^{\theta_{p}} \gamma_{p}\left(a_{k}\right)}{\left(p^{k}+n\right)^{\theta_{p}}} .
$$

Define

$$
f(n)=\frac{\frac{2+a_{k}}{2}\left(\frac{p(p+1)}{2}\right)^{k}+\frac{2+a_{k}}{1+a_{k}} n^{\theta_{p}} \gamma_{p}\left(a_{k}\right)}{\left(p^{k}+n\right)^{\theta_{p}}}
$$

as a function of the continuous variable $n$ (we are interested in showing the inequality of Lemma 5 only for integral values of $n$ but to do this we will think of the bounding function $f$ as a function of a continuous variable so we can use the Calculus). Differentiating gives

$$
\frac{d f}{d n}=\frac{\frac{2+a_{k}}{1+a_{k}} \gamma_{p}\left(a_{k}\right) \theta_{p} n^{\theta_{p}-1} p^{k}-\theta_{p} \frac{2+a_{k}}{2}\left(\frac{p(p+1)}{2}\right)^{k}}{\left(p^{k}+n\right)^{\theta_{p}+1}} .
$$

Setting the numerator equal to zero and cancelling common factors we get

SO

$$
\frac{\gamma_{p}\left(a_{k}\right)}{1+a_{k}} n^{\theta_{p}-1}=\frac{1}{2}\left(\frac{p+1}{2}\right)^{k}
$$

$$
n=\left(\frac{1+a_{k}}{2 \gamma_{p}\left(a_{k}\right)}\right)^{1 /\left(\theta_{p}-1\right)}\left(\left(\frac{p+1}{2}\right)^{1 /\left(\theta_{p}-1\right)}\right)^{k}=\left(\frac{1+a_{k}}{2 \gamma_{p}\left(a_{k}\right)}\right)^{1 /\left(\theta_{p}-1\right)} p^{k} .
$$


As there is only one critical point for $n \geq 0$ and it is a relative minimum as is easily checked by, for example, the Second Derivative Test, it is an absolute minimum for $f(n), n \geq 0$.

Using the definition of $f(n)$ we get

$$
\begin{aligned}
\frac{F_{p}\left(p^{k}+n\right)}{\left(p^{k}+n\right)^{\theta_{p}}}>f(n) \geq f\left(\left(\frac{1+a_{k}}{2 \gamma_{p}\left(a_{k}\right)}\right)^{1 /\left(\theta_{p}-1\right)} p^{k}\right) \\
=\frac{\frac{2+a_{k}}{2}\left(\frac{p(p+1)}{2}\right)^{k}+\frac{2+a_{k}}{1+a_{k}} \gamma_{p}\left(a_{k}\right)\left(p^{\theta_{p}}\right)^{k}\left(\frac{1+a_{k}}{2 \gamma_{p}\left(a_{k}\right)}\right)^{\theta_{p} /\left(\theta_{p}-1\right)}}{\left(p^{k}+p^{k}\left(\frac{1+a_{k}}{2 \gamma_{p}\left(a_{k}\right)}\right)^{1 /\left(\theta_{p}-1\right)}\right)^{\theta_{p}}} .
\end{aligned}
$$

Since $p^{\theta_{p}}=p(p+1) / 2$ we get

$$
\begin{aligned}
\frac{F_{p}\left(p^{k}+n\right)}{\left(p^{k}+n\right)^{\theta_{p}}}> & \frac{2+a_{k}}{2} \cdot \frac{1+\frac{2 \gamma_{p}\left(a_{k}\right)}{1+a_{k}}\left(\frac{1+a_{k}}{2 \gamma_{p}\left(a_{k}\right)}\right)^{\theta_{p} /\left(\theta_{p}-1\right)}}{\left(1+\left(\frac{1+a_{k}}{2 \gamma_{p}\left(a_{k}\right)}\right)^{1 /\left(\theta_{p}-1\right)}\right)^{\theta_{p}}} \\
& =\frac{2+a_{k}}{2} \cdot \frac{1+\left(\frac{1+a_{k}}{2 \gamma_{p}\left(a_{k}\right)}\right)^{1 /\left(\theta_{p}-1\right)}}{\left(1+\left(\frac{1+a_{k}}{2 \gamma_{p}\left(a_{k}\right)}\right)^{1 /\left(\theta_{p}-1\right)}\right)^{\theta_{p}}} \\
& =\frac{2+a_{k}}{2}\left(1+\left(\frac{1+a_{k}}{2 \gamma_{p}\left(a_{k}\right)}\right)^{1 /\left(\theta_{p}-1\right)}\right)^{1-\theta_{p}} .
\end{aligned}
$$

The next lemma will allow us to get a lower bound on $F_{p}(n) / n^{\theta_{p}}$ where $n$ has $k+1$ digits base $p$ given a lower bound on $F_{p}(m) / m^{\theta_{p}}$ for all $m$ with at most $k$ digits base $p$.

Lemma 6. For $n=p^{k+r+1}+a_{k} p^{k+r}+\ldots+a_{1} p^{r+1}+b$ with $0 \leq b=$ $b_{r} p^{r}+\ldots+b_{1} p+b_{0}<p^{r+1}$ and for

$$
A=\left(\frac{p(p+1)}{2}\right)^{k+1}+2 \sum_{i=1}^{k}\left(\left(\frac{p(p+1)}{2}\right)^{i} \frac{a_{i}\left(a_{i}+1\right)}{2} \prod_{j=i+1}^{k}\left(a_{j}+1\right)\right)
$$

and

$$
B=2 \gamma_{p}\left(b_{r}\right) \prod_{i=1}^{k}\left(a_{i}+1\right)
$$


with $\gamma_{p}$ as in the previous lemma, we have

$$
\begin{aligned}
\left.\frac{F_{p}\left(p^{k+r+1}+a_{k} p^{k+r}+\ldots+a_{1} p^{r+1}+b\right)}{\left(p^{k+r+1}+a_{k} p^{k+r}+\ldots\right.}+a_{1} p^{r+1}+b\right)^{\theta_{p}} & \\
> & \frac{A p^{r}}{n-b}\left(\frac{n-b}{p^{r}}+\left(\frac{A p^{r}}{(n-b) B}\right)^{1 /\left(\theta_{p}-1\right)}\right)^{1-\theta_{p}} .
\end{aligned}
$$

Pr o of. Using Lemmas 1 and 2 we have

$$
\begin{aligned}
\frac{F_{p}\left(p^{k+r+1}+a_{k} p^{k+r}+\ldots+a_{1} p^{r+1}+b\right)}{\left(p^{k+r+1}+a_{k} p^{k+r}+\ldots+a_{1} p^{r+1}+b\right)^{\theta_{p}}} & \left(\frac{p(p+1)}{2}\right)^{k+r+1}+2 \sum_{i=1}^{k}\left(\frac{a_{i}\left(1+a_{i}\right)}{2}\left(\frac{p(p+1)}{2}\right)^{r+i} \prod_{j>i}\left(1+a_{j}\right)\right) \\
= & \frac{2\left(\prod_{i=1}^{k+r+1}+a_{k} p^{k+r}+\ldots+a_{1} p^{r+1}+b\right)^{\theta_{p}}}{\left.\left(1+a_{i}\right)\right) F_{p}(b)} \\
& +\frac{\left(\frac{p(p+1)}{2}\right)^{k+r+1}+2 \sum_{i=1}^{k}\left(\frac{a_{i}\left(1+a_{i}\right)}{2}\left(\frac{p(p+1)}{2}\right)^{r+i} \prod_{j>i}\left(1+a_{j}\right)\right)}{\left(p^{k+r+1}+a_{k} p^{k+r}+\ldots+a_{1} p^{r+1}+b\right)^{\theta_{p}}} \\
> & \frac{2\left(\prod_{i=1}^{k}\left(1+a_{i}\right)\right) \gamma_{p}\left(b_{r}\right) b^{\theta_{p}}}{\left(p^{k+r}+\ldots+a_{1} p^{r+1}+b\right)^{\theta_{p}}} \\
& +\frac{\left.a_{k} p^{k+r}+\ldots+a_{1} p^{r+1}+b\right)^{\theta_{p}}}{\left(p^{k+r+1}+\ldots\right.}
\end{aligned}
$$

Factoring out $p^{r \theta_{p}}$ from the denominator and noting that $p^{\theta_{p}}=p(p+1) / 2$ we define

$$
f(b)=\frac{A+B\left(b / p^{r}\right)^{\theta_{p}}}{\left(p^{k+1}+a_{k} p^{k}+\ldots+a_{1} p^{1}+b / p^{r}\right)^{\theta_{p}}}
$$

so

$$
\frac{F_{p}\left(p^{k+r+1}+a_{k} p^{k+r}+\ldots+a_{1} p^{r+1}+b\right)}{\left(p^{k+r+1}+a_{k} p^{k+r}+\ldots+a_{1} p^{r+1}+b\right)^{\theta_{p}}}>f(b) .
$$

Treating $f(b)$ as a continuous function of $b$ we find the only critical point for $f(b)$ is a relative minimum at

$$
b=\left(\frac{A p^{r \theta_{p}}}{B(n-b)}\right)^{1 /\left(\theta_{p}-1\right)} .
$$


Therefore

$$
\begin{aligned}
& \frac{F_{p}\left(p^{k+r+1}+a_{k} p^{k+r}+\ldots+a_{1} p^{r+1}+b\right)}{\left(p^{k+r+1}+a_{k} p^{k+r}+\ldots+a_{1} p^{r+1}+b\right)^{\theta_{p}}}>f(b) \\
& \geq f\left(\left(\frac{A p^{r \theta_{p}}}{B(n-b)}\right)^{1 /\left(\theta_{p}-1\right)}\right) \\
& =\frac{A+B\left(\frac{A p^{r}}{B(n-b)}\right)^{\theta_{p} /\left(\theta_{p}-1\right)}}{\left(\frac{n-b}{p^{r}}+\left(\frac{A p^{r}}{B(n-b)}\right)^{1 /\left(\theta_{p}-1\right)}\right)^{\theta_{p}}} \\
& =\frac{A p^{r}}{n-b}\left(\frac{n-b}{p^{r}}+\left(\frac{A p^{r}}{B(n-b)}\right)^{1 /\left(\theta_{p}-1\right)}\right)^{1-\theta_{p}} .
\end{aligned}
$$

Since $(n-b) / p^{r}=p^{k+1}+a_{k} p^{k}+\ldots+a_{1} p$ it is obvious that this lower bound does not depend on $r$.

3. Values of $\beta_{p}$ for small $p$. Using Lemmas 3,5 and 6 and a fair bit of machine computation it is possible to approximate the values of $\beta_{p}$ to any number of desired decimal places. In the theorem below we give the values of $\beta_{p}, p<20$, to six decimal places. In the next section we will get some bounds on $\beta_{p}$ and use these to investigate the nature of $\beta_{p}$ for large $p$.

\section{Theorem 1.}

$$
\begin{gathered}
\beta_{3}=.774281 \ldots, \quad \beta_{5}=.758226 \ldots, \quad \beta_{7}=.749117 \ldots, \quad \beta_{11}=.736495 \ldots, \\
\beta_{13}=.732663 \ldots, \quad \beta_{17}=.727582 \ldots, \quad \beta_{19}=.725754 \ldots
\end{gathered}
$$

Proof. We will prove this for $p=5$. The other proofs are similar. First note that

and by Lemma 3 ,

$$
\frac{F_{5}(2929687)}{2929687^{\theta_{5}}}=.758226 \ldots
$$

$$
\frac{F_{5}\left(5^{k} \cdot 2929687\right)}{\left(5^{k} \cdot 2929687\right)^{\theta_{5}}}=\frac{F_{5}(2929687)}{2929687^{\theta_{5}}} .
$$

This means

$$
\beta_{5} \leq \frac{F_{5}(2929687)}{2929687^{\theta_{5}}}=.758226 \ldots
$$

To complete the proof we must show $F_{5}(n) / n^{\theta_{5}} \geq .758226$ for all $n$. Note that if $F_{5}(n) / n^{\theta_{5}} \geq .758226$ for all $1 \leq n<2 \cdot 5^{k}$, then Lemma 5 says

$$
\frac{F_{5}(n)}{n^{\theta_{5}}} \geq .802517 \ldots \quad \text { for all } 2 \cdot 5^{k} \leq n<3 \cdot 5^{k}
$$




$$
\begin{array}{ll}
\frac{F_{5}(n)}{n^{\theta_{5}}} \geq .850443 \ldots & \text { for all } 3 \cdot 5^{k} \leq n<4 \cdot 5^{k}, \\
\frac{F_{5}(n)}{n^{\theta_{5}}} \geq .895474 \ldots & \text { for all } 4 \cdot 5^{k} \leq n<5^{k+1} .
\end{array}
$$

Therefore if $F_{5}(n) / n^{\theta_{5}} \geq .758226$ for all $1 \leq n<2 \cdot 5^{k}$ then $F_{5}(n) / n^{\theta_{5}} \geq$ .758226 for all $1 \leq n<5^{k+1}$. We would like to show this is true for all $n$ so we will extend the region where $F_{5}(n) / n^{\theta_{5}} \geq .758226$ to $1 \leq n<2 \cdot 5^{k+1}$ and induct on $k$.

By Lemma 6 , if $n=5^{k+1}+b, b=b_{k} 5^{k}+\ldots+b_{1} 5+b_{0}$, then

$$
\begin{array}{ll}
\frac{F_{5}(n)}{n^{\theta_{5}}}>.784931 \ldots>.758226 & \text { if } b_{k}=4, \\
\frac{F_{5}(n)}{n^{\theta_{5}}}>.772610 \ldots>.758226 & \text { if } b_{k}=3, \\
\frac{F_{5}(n)}{n^{\theta_{5}}}>.758226 \ldots>.758226 & \text { if } b_{k}=2, \\
\frac{F_{5}(n)}{n^{\theta_{5}}}>.743618 \ldots<.758226 & \text { if } b_{k}=0,1 .
\end{array}
$$

Using more information we get better bounds when $b_{k}=0,1$. If $b_{k}=1$ then $n=5^{k+1}+5^{k}+b, b=b_{k-1} 5^{k-1}+\ldots+b_{1} 5+b_{0}$. Using Lemma 6 we get

$$
\begin{array}{ll}
\frac{F_{5}(n)}{n^{\theta_{5}}}>.780155 \ldots>.758226 & \text { if } b_{k-1}=4, \\
\frac{F_{5}(n)}{n^{\theta_{5}}}>.778535 \ldots>.758226 & \text { if } b_{k-1}=3, \\
\frac{F_{5}(n)}{n^{\theta_{5}}}>.776573 \ldots>.758226 & \text { if } b_{k-1}=2, \\
\frac{F_{5}(n)}{n^{\theta_{5}}}>.774498 \ldots>.758226 & \text { if } b_{k-1}=0,1 .
\end{array}
$$

If $b_{k}=0$ then $n=5^{k+1}+b, b=b_{k-1} 5^{k-1}+\ldots+b_{1} 5+b_{0}$. Using Lemma 6 we get

$$
\begin{array}{ll}
\frac{F_{5}(n)}{n^{\theta_{5}}}>.772683 \ldots>.758226 & \text { if } b_{k-1}=4, \\
\frac{F_{5}(n)}{n^{\theta_{5}}}>.768335 \ldots>.758226 & \text { if } b_{k-1}=3, \\
\frac{F_{5}(n)}{n^{\theta_{5}}}>.763119 \ldots>.758226 & \text { if } b_{k-1}=2, \\
\frac{F_{5}(n)}{n^{\theta_{5}}}>.757661 \ldots<.758226 & \text { if } b_{k-1}=0,1 .
\end{array}
$$

Our two exceptional cases are thus $n=5^{k+1}+5^{k-1}+b, n=5^{k+1}+b$, $1 \leq b<5^{k-1}$. We could continue to apply Lemma 6 but at each step we 
would still get two exceptional cases; however, these two values for $n$ can be shown to satisfy the requisite inequality in another way:

$$
\begin{aligned}
\frac{F_{5}\left(5^{k+1}+5^{k-1}+b\right)}{\left(5^{k+1}+5^{k-1}+b\right)^{\theta_{5}}} & >\frac{F_{5}\left(5^{k+1}+5^{k-1}\right)}{\left(5^{k+1}+2 \cdot 5^{k-1}\right)^{\theta_{5}}} \\
& =\frac{F_{5}(26)}{27^{\theta_{5}}}=.886348 \ldots>.758226
\end{aligned}
$$

and similarly,

$$
\frac{F_{5}\left(5^{k+1}+b\right)}{\left(5^{k+1}+b\right)^{\theta_{5}}}>\frac{F_{5}\left(5^{k+1}\right)}{\left(5^{k+1}+5^{k-1}\right)^{\theta_{5}}}=\frac{F_{5}(25)}{26^{\theta_{5}}}=.936137 \ldots>.758226 .
$$

Summarizing: in all cases $F_{5}(n) / n^{\theta_{5}}>.758226$ for $1 \leq n<2 \cdot 5^{k+1}$ provided it is so for all $1 \leq n<2 \cdot 5^{k}$. To start our induction we need to check all $n$ with $1 \leq n<10(k=1)$ since we used at most two digits before the $5^{k+1}$ to establish the bound for $1 \leq n<2 \cdot 5^{k+1}$. A quick check shows $F_{5}(n) / n^{\theta_{5}}>.758226$ for all $1 \leq n<10$.

4. Behavior of $\beta_{p}$ for large $p$. Another problem of interest is to investigate the behavior of $\beta_{p}$ as $p$ grows. A first step in this direction is the following bounding theorem. It improves on Stein [4], $\beta_{p}>1 / p$, and on the easily obtainable bound $\beta_{p}>2 /(p(p+1))$ mentioned in Volodin [7].

TheOREM 2. For all primes $p$,

$$
\left(1-2^{1 /\left(1-\theta_{p}\right)}\right)^{\theta_{p}-1} \leq \beta_{p}<\frac{3-\theta_{p}}{2\left(2-\theta_{p}\right)^{2-\theta_{p}}} .
$$

Pr o of. Since $\beta_{p}=\liminf _{n \rightarrow \infty} F_{p}(n) / n^{\theta_{p}}$ Lemma 3 says that $\beta_{p} \leq q_{0}(p)$ for $q_{0}(p)=\min _{n=1, \ldots, p} F_{p}(n) / n^{\theta_{p}}$. Define

$$
f_{p}(x)=\frac{x(x+1) / 2}{x^{\theta_{p}}} .
$$

Differentiating $f_{p}(x)$ we get

$$
\frac{d f_{p}}{d x}=\frac{\left(2-\theta_{p}\right) x+\left(1-\theta_{p}\right)}{2 x^{\theta_{p}}}
$$

so our lone critical point (a minimum) is at

$$
x_{\min }=\frac{\theta_{p}-1}{2-\theta_{p}} \text {. }
$$

Since there is an integer in $\left[x_{\min }, 1+x_{\min }\right)$ we know $q_{0}(p)<f_{p}\left(1+x_{\min }\right)$. This leads to

$$
\beta_{p}<f_{p}\left(1+x_{\min }\right)=\frac{3-\theta_{p}}{2\left(2-\theta_{p}\right)^{2-\theta_{p}}} .
$$

This establishes the upper bound. 
We prove the lower bound by induction on $k$. Suppose

$$
\frac{F_{p}(n)}{n^{\theta_{p}}}>\left(1-2^{1 /\left(1-\theta_{p}\right)}\right)^{\theta_{p}-1} \quad \text { for all } 1 \leq n<p^{k} .
$$

Then

$$
\begin{aligned}
\frac{F_{p}\left(a p^{k}+n\right)}{\left(a p^{k}+n\right)^{\theta_{p}}} & =\frac{\frac{a(a+1)}{2}\left(\frac{p(p+1)}{2}\right)^{k}+(a+1) F_{p}(n)}{\left(a p^{k}+n\right)^{\theta_{p}}} \\
& >\frac{\frac{a(a+1)}{2}\left(\frac{p(p+1)}{2}\right)^{k}+(a+1)\left(1-2^{1 /\left(1-\theta_{p}\right)}\right)^{\theta_{p}-1} n^{\theta_{p}}}{\left(a p^{k}+n\right)^{\theta_{p}}} .
\end{aligned}
$$

We define

$$
f_{p}(n)=\frac{\frac{a(a+1)}{2}\left(\frac{p(p+1)}{2}\right)^{k}+(a+1)\left(1-2^{1 /\left(1-\theta_{p}\right)}\right)^{\theta_{p}-1} n^{\theta_{p}}}{\left(a p^{k}+n\right)^{\theta_{p}}} .
$$

Treating $f_{p}$ as a continuous function of $n$ we get a single critical point,

$$
n_{\min }=p^{k} \frac{2^{1 /\left(1-\theta_{p}\right)}}{1-2^{1 /\left(1-\theta_{p}\right)}} .
$$

Evaluating $f_{p}$ at this point we have

$$
\frac{F_{p}\left(a p^{k}+n\right)}{\left(a p^{k}+n\right)^{\theta_{p}}}>f_{p}\left(n_{\min }\right)=\frac{a+1}{2}\left(a+\frac{2^{1 /\left(1-\theta_{p}\right)}}{1-2^{1 /\left(1-\theta_{p}\right)}}\right)^{1-\theta_{p}} .
$$

To determine which value of $a$ minimizes this expression we differentiate and find the only critical point (a minimum) is at

$$
a_{\min }=\frac{\theta_{p}-\frac{1}{1-2^{1 /\left(1-\theta_{p}\right)}}}{2-\theta_{p}} .
$$

It can be shown that $a_{\min }<1$ so among the values $1, \ldots, p-1$, the minimum for $f_{p}\left(n_{\min }\right)$ occurs at $a=1$, i.e.,

$$
\frac{F_{p}\left(a p^{k}+n\right)}{\left(a p^{k}+n\right)^{\theta_{p}}}>\frac{1+1}{2}\left(1+\frac{2^{1 /\left(1-\theta_{p}\right)}}{1-2^{1 /\left(1-\theta_{p}\right)}}\right)^{1-\theta_{p}}=\left(1-2^{1 /\left(1-\theta_{p}\right)}\right)^{\theta_{p}-1}
$$

Theorem 2 can be used to say what happens to $\beta_{p}$ for large values of $p$.

THEOREM 3.

$$
\lim _{p \rightarrow \infty} \beta_{p}=.5 .
$$

Proof. By Theorem 2 and the Squeeze Theorem it is enough to show

$$
\lim _{p \rightarrow \infty}\left(1-2^{1 /\left(1-\theta_{p}\right)}\right)^{\theta_{p}-1}=.5
$$


and

These are both easily verified.

$$
\lim _{p \rightarrow \infty} \frac{3-\theta_{p}}{2\left(2-\theta_{p}\right)^{2-\theta_{p}}}=.5
$$

5. Conjectures. In [2] it was conjectured that $\beta_{2}=\lim _{r \rightarrow \infty} q_{2}(r)$ (where $q_{r}=F_{2}\left(n_{r}\right) / n_{r}^{\theta_{2}}$ for $n_{0}=1, n_{r}=n_{r-1} \pm 1$ for $r \geq 1$, with sign chosen to minimize $q_{r}$ ). To generalize this conjecture for an odd prime $p$, choose $n_{p}(0), n_{p}(1)$ to minimize $F_{p}(n) / n^{\theta_{p}}$ on $\{1, \ldots, p\}$ and $\left\{p+1, \ldots, p^{2}\right\}$ respectively, $n_{p}(r)=p n_{p}(r-1) \pm(p \pm 1) / 2$ for $r \geq 2$ with signs chosen independently to minimize $F_{p}\left(n_{p}(r)\right) / n_{p}(r)^{\theta_{p}}$. Let $q_{p}(r)=F_{p}\left(n_{p}(r)\right) / n_{p}(r)^{\theta_{p}}$.

Conjecture 1.

$$
\beta_{p}=\lim _{r \rightarrow \infty} q_{p}(r) .
$$

It was in using such a sequence for $p=5$ that led to our checking $F_{5}(n) / n^{\theta_{5}}$ at $n=2929687$ in the proof of Theorem 1 . Similarly such sequences for $p<20$ give the values of $\beta_{p}$ at least to 6 decimal places. For the primes $p=3,5,7,17,19$ the choice always seems to be $+(p-1) / 2$ but the author has no proof of this at present. More generally, we have Conjecture 2.

Conjecture 2. For $p$ an odd prime there exists $b$ such that

$$
n_{p}(r)=p n_{p}(r-1)+\frac{p-1}{2} \quad \text { for all } r \geq b .
$$

For example the minimum for $F_{11}(n) / n^{\theta_{11}}$ for $11^{k} \leq n<11^{k+1}$ seems to be at $n=1455 \ldots 55_{(11)}$ for all $k \geq 3$. The base $p$ expansions for the minima for $p=5,7,11$ lead us to Conjecture 3 .

Conjecture 3 .

$$
\beta_{5}=\left(\frac{3}{2}\right)^{1-\theta_{5}}, \quad \beta_{7}=\left(\frac{3}{2}\right)^{1-\theta_{7}}, \quad \beta_{11}=\frac{59 / 44}{(31 / 22)^{\theta_{11}}} .
$$

By Theorem 1 the values in Conjecture 3 are correct to at least six decimal places. Volodin [7] conjectured $\beta_{3}=2^{\log _{3} 2-1}=(3 / 2)^{1-\theta_{3}}$ but conjectured incorrect values for $\beta_{5}$ and $\beta_{7}$. The simple form for the liminf, $\beta_{p}=(3 / 2)^{1-\theta_{p}}$, can hold for at most finitely many $p$ by Theorem 2 and the fact that $\lim _{p \rightarrow \infty} \theta_{p}=2$.

\section{References}

[1] N. J. Fine, Binomial coefficients modulo a prime, Amer. Math. Monthly 54 (1947), 589-592.

[2] H. Harborth, Number of odd binomial coefficients, Proc. Amer. Math. Soc. 62 (1977), 19-22. 
[3] E. E. Kummer, Über die Ergänzungssätze zu den allgemeinen Reciprocitätsgesetzen, J. Reine Angew. Math. 44 (1852), 93-146.

[4] A. H. Stein, Binomial coefficients not divisible by a prime, in: Number Theory, Lecture Notes in Math. 1383, Springer, New York, 1989, 170-177.

[5] K. B. Stolarsky, Digital sums and binomial coefficients, Notices Amer. Math. Soc. 22 (1975), A-669.

[6] - Power and exponential sums of digital sums related to binomial coefficient parity, SIAM J. Appl. Math. 32 (1977), 717-730.

[7] N. A. Volodin, Number of multinomial coefficients not divisible by a prime, Fibonacci Quart. 32 (1994), 402-406.

Department of Mathematics

SUNY College at Brockport

Brockport, New York 14420

U.S.A.

E-mail: bwilson@acspr1.acs.brockport.edu

Received on 10.6.1996

and in revised form on 20.5.1997 